\title{
Overexpression of the mitochondrial chaperone tumor necrosis factor receptor-associated protein 1 is associated with the poor prognosis of patients with colorectal cancer
}

\author{
CHANG GAO $^{1 *}$, MIN LI $^{1 *}$, AN-LI JIANG ${ }^{2}$, RUI SUN ${ }^{1}$, HONG-LIN JIN ${ }^{3}$, \\ HUA-WEI GUI ${ }^{4}$, FEI XIAO ${ }^{1}$, XIANG-WU DING ${ }^{1}$, ZHEN-MING FU ${ }^{5}$ and JUE-PING FENG ${ }^{1}$
}

${ }^{1}$ Department of Oncology, PuAi Hospital of Tongji Medical College, Huazhong University of Science and Technology, Wuhan, Hubei 430034; ${ }^{2}$ Department of Clinical Medicine, Medical College, Wuhan University of Science and Technology, Wuhan, Hubei 430065; ${ }^{3}$ Cancer Center, Union Hospital, Tongji Medical College, Huazhong University of Science and Technology, Wuhan, Hubei 430022; ${ }^{4}$ Department of Pathology, PuAi Hospital of Tongji Medical College, Huazhong University of Science and Technology, Wuhan, Hubei 430034; ${ }^{5}$ Department of Oncology, Renmin Hospital of Wuhan University, Wuhan, Hubei 430060, P.R. China

Received November 4, 2016; Accepted October 26, 2017

DOI: $10.3892 / 01.2018 .8042$

\begin{abstract}
Tumor necrosis factor receptor-associated protein-1 (TRAP-1), a mitochondrial chaperone, contributes significantly to the progression of cancer. However, the understanding of its involvement in the clinicopathological characteristics and prognosis of colorectal cancer (CRC) remains limited. The aim of the present study was to assess the significance of TRAP-1 expression in CRC. The expression of TRAP-1 was evaluated in corresponding cancerous, paracancerous, lymph node and distant metastatic tissues of 256 cases of CRC by immunohistochemistry. The associations between TRAP-1 expression and the clinicopathological parameters and survival rates of patients was assessed. Out of 256 patients with CRC, TRAP-1 expression was detected in 203 (79.3\%). TRAP-1 expression was significantly increased in cancerous tissue compared with that in corresponding paracancerous tissues $(\mathrm{P}<0.001)$. Overexpression of TRAP-1 was significantly associated with differentiation $(\mathrm{P}=0.011)$, depth of invasion $(\mathrm{P}=0.006)$, lymph node metastasis $(\mathrm{P}<0.001)$ and tumor-node-metastasis stage $(\mathrm{P}<0.001)$. In patients with high TRAP-1 expression, the 5-year overall survival (OS) rate was $38.0 \%$, in contrast to $56.5 \%$ in patients with low TRAP-1 expression $(\mathrm{P}=0.003)$. Similarly, the 5 -year progression-free survival (PFS) was $26.6 \%$ for patients
\end{abstract}

Correspondence to: Professor Jue-Ping Feng, Department of Oncology, PuAi Hospital of Tongji Medical College, Huazhong University of Science and Technology, 76 Jiefang Street, Wuhan, Hubei 430034, P.R. China

E-mail: fengjuepingpuai@163.com

\section{*Contributed equally}

Key words: tumor necrosis factor receptor-associated protein 1, colorectal cancer, prognosis, immunohistochemistry, biomarker with high TRAP-1 expression and 53.3\% for patients with low TRAP-1 expression $(\mathrm{P}<0.001)$. Multivariate analyses indicated the TRAP-1 expression is an independent prognostic factor for poorer OS $[\mathrm{P}=0.015$; hazard ratio $(\mathrm{HR}), 1.914]$ and $\mathrm{PFS}$ $(\mathrm{P}<0.001$; HR, 2.534). Thus, TRAP-1 may serve as a potential biomarker for predicting the prognosis of patients with CRC. Specifically, overexpression of TRAP-1 may predict progression and poor survival in cases of CRC.

\section{Introduction}

Colorectal cancer (CRC) is the third leading cause of cancer-associated mortality worldwide (1). Recurrence and metastasis are the major causes of mortality in patients with CRC $(2,3)$. Although screening, surgical techniques and adjuvant chemotherapy for patients with CRC have been developed to achieve a significant improvement in survival time $(4,5)$, the 5 -year overall survival (OS) rate of CRC remains at $\sim 65 \%$ (6). The stage of CRC at the time of diagnosis is a key factor for deciding the therapeutic regimen and assessing prognosis. However, at the time of diagnosis, the majority of patients exhibit intermediate- or late-stage disease $(7,8)$. Therefore, efficient diagnostic and predictive biomarkers are required for early-stage diagnosis in order to improve the prognosis of patients with CRC.

Themitochondrial tumor necrosis factor receptor-associated protein-1 (TRAP-1) is one of the 90 isoforms of heat shock protein (HSP) and is also termed HSP75 $(9,10)$. TRAP-1 has been demonstrated to serve an effective function in stabilizing cellular homeostasis and regulating mitochondrial integrity $(11,12)$. By antagonizing cyclophilin D (CypD), TRAP-1 serves a cytoprotective function for cells responding to apoptotic stimuli and enables tumor cell survival in a hostile environment (13). It has been reported that TRAP-1 protects cancer cells from apoptosis by blocking mitochondrial effects (14). Additionally, increasing evidence has demonstrated that increased TRAP-1 expression is observed 
in cancerous tissues of the breast, lung, prostate and pancreas as compared with the corresponding normal tissues (15-17). A previous study also demonstrated that TRAP-1 expression level is associated with lymph node metastasis in CRC (18). These aforementioned results indicate that TRAP-1 is a potential candidate biomarker for breast, lung and prostate cancer, as well as CRC, and may be a cancer-specific therapeutic target. However, only a limited number of studies have evaluated the prognostic relevance of TRAP-1 expression based on clinical data of CRC.

The aim of the present study was to investigate the association between TRAP-1 expression and the clinicopathological features of patients with CRC, including tumor-node-metastasis (TNM) stage, degree of pathological differentiation, and lymph node metastasis, as well as to demonstrate any clinical significance of TRAP-1 expression in CRC prognosis.

\section{Materials and methods}

Patients and follow-up. A total of 256 patients diagnosed with $\mathrm{CRC}$, and who underwent surgical resection at $\mathrm{PuAi}$ Hospital of Tongji Medical College, Huazhong University of Science and Technology of Wuhan (Wuhan, China) between 1 January 2004 and 31 December 2009, were investigated in the present study. None of the patients received neoadjuvant chemotherapy or radiotherapy prior to surgery. Among the 256 patients, 226 patients received 5-fluorouracil (5-FU)-based chemotherapy following surgical resection, including 5-FU alone (5-FU/leucovorin; $500 \mathrm{mg} / \mathrm{m}^{2} 5$-FU on days $1-5$ and $200 \mathrm{mg} / \mathrm{m}^{2}$ leucovorin on days $1-5$, capecitabine (a 5-FU derivative) alone $\left(1,250 \mathrm{mg} / \mathrm{m}^{2}\right.$, twice daily on days $\left.1-14\right)$, and $5-\mathrm{FU}$ combined with oxaliplatin $\left(500 \mathrm{mg} / \mathrm{m}^{2} 5-\mathrm{FU}\right.$ on days $1-5$, and $130 \mathrm{mg} / \mathrm{m}^{2}$ oxaliplatin on day 1). These 226 cases underwent a 21-day cycle chemotherapy for 8 cycles.

By December 31st, 2014, at the end of follow-up, the median follow-up time was 60 months (range, 1-60 months). The outpatient and inpatient medical records, physical exams, complete blood biochemical detection, serum carcinoembryonic antigen and carbohydrate antigen 19-9 measurements, ultrasound and endoscopic examinations, as well as computed tomography scans, were conducted to monitor the status of patients every 3-6 months. Differentiation grading and TNM classification for enrolled patients with colorectal cancer were based on the 7th American Joint Committee on Cancer TNM staging system (19).

The OS time was defined as the interval from the date of CRC surgery until mortality from any cause. Progression-free survival (PFS) was defined as the interval from a particular treatment to the first evidence of progression or recurrence, or to disease-associated mortality.

All procedures used in the present study involving human participants were approved by the Ethics Committee of PuAi Hospital of Tongji Medical College, Huazhong University of Science and Technology, and were performed in accordance with the 1964 Declaration of Helsinki and its later amendments or comparable ethical standards. Informed consent was obtained from all individual participants included in the study.

Tissue specimens. Samples of 256 paired CRC tissues and paracancerous tissues were collected and examined. To assess metastatic CRC, 33 metastatic CRC tissue samples from 25 cases were selected for analysis (multiple-site metastases were present in 8 cases), including 4 cases metastasized to the pelvis, 4 cases metastasized to the liver, 2 cases metastasized to the lung, 2 cases metastasized to the bladder, 15 cases metastasized to the peritoneum and 6 cases metastasized to other organs, such as the ovaries. To assess lymph node metastasis, $>12$ lymph nodes were obtained and studied. A total of 53 cases of patients who underwent colonoscopy were also examined, including 25 cases of colorectal adenoma and 28 cases of colorectal high-grade intraepithelial neoplasia. All specimens were fixed in $10 \%$ formalin for $24 \mathrm{~h}$ at room temperature and embedded in paraffin.

Immunohistochemistry (IHC) for TRAP-1. Sections from formalin-fixed, paraffin embedded human colorectal tissues measuring 3- $\mu \mathrm{m}$ thick were sectioned on slides. The sections were then deparaffinized at room temperature with xylene, hygradated with ethyl alcohol in the descending gradients (100, 95 and $75 \%)$ and washed in distilled water several times. Antigen retrieval was conducted using Heat Induced Epitope Retrieval with a pressure-cooker for $10 \mathrm{~min}$ after the pressure starting beating in $0.01 \mathrm{M}$ citrate antigen retrieval solution ( $\mathrm{pH}$ 6.0). Subsequent to cooling to room temperature, those sections were processed with $0.3 \% \mathrm{H}_{2} \mathrm{O}_{2}$ methanol for $10 \mathrm{~min}$ to remove endogenous peroxidase activity at room temperature. IHC staining for TRAP-1 was conducted as previously described $(20,21)$. Briefly, slides were incubated with a monoclonal rabbit anti-TR AP-1 primary antibody (clone EPR5381, cat. no. ab109323, dilution 1:200; Abcam, Cambridge, MA, USA; ) at $4^{\circ} \mathrm{C}$ overnight, followed by incubation with a horseradish peroxidase-labeled goat anti-rabbit IgG secondary antibody (cat. no. A-11035; dilution 1:500; Invitrogen; Thermo Fisher Scientific, Inc., Waltham, MA, $\mathrm{USA}$; ) at $37^{\circ} \mathrm{C}$ for $2 \mathrm{~h}$. Slides were scored by two experienced pathologists according to intensity and proportion of immunostaining using an upright optical microscope (Olympus Corporation, Tokyo, Japan). Immunostaining was assessed in 5 high-powered fields at magnification, x 200. Cytoplasmic staining of epithelial cells was considered positive. The intensity of immunohistochemical staining (0, negative; 1 , weak; 2 , intermediate; 3 , strong) and the percentage of positive cells $(0,<5 \%$ positive cells; $1,5-25 \%$ positive cells; $2,26-50 \%$ positive cells; $3,>50 \%$ positive cells) were evaluated. As for calculating the percentage of positive cells of each slide, 5 fields of high power lens were selected for observation. The final score for each section was assessed by multiplying the scores of staining intensity and percentage of positive cells. The immunohistochemical staining score was calculated by taking the average of the staining scores assigned by each pathologist, according to the formula [(score 1: Percentage of area stained $x$ staining intensity) + (score 2: Percentage of area stained $\mathrm{x}$ staining intensity)]/2, as previously described (18). The two scores were evaluated by two pathologists respectively. For statistical analysis, tissue specimens were divided into the following four groups according to their immunohistochemical score: Negative (staining score, 0); positive (staining score, 1-12); low (staining score, 1-4); intermediate (staining score, 5-8); and high (staining score, 9-12). 
Statistical analysis. Statistical analyses were performed using SPSS software (version 13.0; SPSS Inc., Chicago, IL, USA). For comparative analysis of qualitative variables, Pearson's $\chi^{2}$ test and Fisher's exact test were used to evaluate the association between the expression levels of TRAP-1 and clinical factors. OS and PFS were analyzed using Kaplan-Meier Plot, and the log-rank test was performed to detect the significance of prognostic factors. Cox's proportional hazards model was applied to identify independent predictive factors for survival. All P-values were two-sided, and $\mathrm{P}<0.05$ were considered to indicate a statistically significant difference.

\section{Results}

Clinical characteristics of patients with CRC. In the 256 cases of CRC analyzed, patient age at diagnosis ranged from 29 -80 years (mean $\pm \mathrm{SD}, 61.9 \pm 11.0$ years). Among these cases, 122 patients $(47.7 \%)$ were male and $134(52.3 \%)$ patients were female. The primary tumor site was the colon in $151(59.0 \%)$ patients and the rectum in $105(41.0 \%)$ patients. Lymph node metastases were present in 117 (45.7\%) patients (Table I).

Expression of TRAP-1 in CRC and its clinicopathological association. TRAP-1 expression was investigated in paired cancerous and paracancerous tissue sections in 256 patients with CRC. Positive expression was observed in 203/256 (79.3\%) cancerous tissue samples and in 25/256 (9.8\%) paracancerous tissue samples. In positive samples, TRAP-1 was primarily localized in the cytoplasm of colorectal epithelial cells. The positive expression levels were significantly increased in cancerous tissues compared with paracancerous tissue samples $(\mathrm{P}<0.001$; Fig. 1A). A total of 77 (30.1\%) samples of cancerous tissue displayed high TRAP-1 expression, 86 (33.6\%) samples exhibited intermediate TRAP-1 expression, 40 (15.6\%) samples exhibited low TRAP-1 expression, and $53(20.7 \%)$ samples exhibited negative TRAP-1 expression.

In the present study, the associations between TRAP-1 expression and clinicopathological features were analyzed. For the purpose of this analysis, the high expression group comprised patients who possessed a TRAP-1 expression score of 9-12 ( $n=77,30.1 \%)$, while all other patients (with intermediate, low or negative TRAP-1 expression) were assigned to the low expression group $(n=179,69.9 \%)$. Clinicopathological features included sex (male vs. female), age ( $<65$ vs. $\geq 65$ years), primary tumor location (colon vs. rectum), TNM stage, [stage I/II (defined as early stage) vs. stage III/IV (defined as advanced stage)], tumor invasion (T1/T2 vs. T3/T4), histodifferentiation (well/moderate vs. poor) and lymph node metastasis (N0 vs. N1/N2). The results demonstrated that TRAP-1 expression levels were associated with the degree of differentiation $(\mathrm{P}=0.011)$, depth of invasion ( $\mathrm{T}$ stage; $\mathrm{P}=0.006)$, lymph node metastasis $(\mathrm{N}$ stage; $\mathrm{P}<0.001)$ and $\mathrm{TNM}$ stage $(\mathrm{P}<0.001$; Table II; Fig. 1B). No significant association was observed between TRAP-1 expression and sex, age or tumor location ( $\mathrm{P}>0.05$; Table II).

In addition, 25 colorectal adenoma tissues and 28 colorectal high-grade intraepithelial neoplasia tissues were analyzed. The results demonstrated weakly positive TRAP-1 expression (staining score, 1-3) in all 28 high-grade intraepithelial neoplasia tissues as well as in 2/25 colorectal adenoma tissues,
Table I. Baseline characteristics of patients with colorectal cancer $(n=256)$.

\begin{tabular}{|c|c|}
\hline Characteristics & $\mathrm{n}(\%)$ \\
\hline \multicolumn{2}{|l|}{ Age, years } \\
\hline$<65$ & $140(54.7)$ \\
\hline$\geq 65$ & $116(45.3)$ \\
\hline \multicolumn{2}{|l|}{ Sex } \\
\hline Male & $122(47.7)$ \\
\hline Female & $134(52.3)$ \\
\hline \multicolumn{2}{|l|}{ Site of primary tumor } \\
\hline Colon & $151(59.0)$ \\
\hline Rectum & $105(41.0)$ \\
\hline \multicolumn{2}{|l|}{$\mathrm{N}$ staging } \\
\hline No & $139(54.3)$ \\
\hline N1-N2 & $117(45.7)$ \\
\hline \multicolumn{2}{|l|}{ TNM stage ${ }^{\mathrm{a}}$} \\
\hline I & $25(9.8)$ \\
\hline II & $113(44.1)$ \\
\hline III & $93(36.3)$ \\
\hline IV & $25(9.8)$ \\
\hline \multicolumn{2}{|l|}{ Surgery } \\
\hline Curative resection intent & $230(89.8)$ \\
\hline Palliative resection & $26(10.2)$ \\
\hline \multicolumn{2}{|l|}{ Chemotherapy } \\
\hline Yes & $226(88.3)$ \\
\hline No & $30(11.7)$ \\
\hline \multicolumn{2}{|l|}{ Differentiation $^{\mathrm{a}}$} \\
\hline Well-differentiated & $58(22.6)$ \\
\hline Moderately differentiated & $129(50.4)$ \\
\hline Poorly differentiated & $69(27.0)$ \\
\hline \multicolumn{2}{|l|}{ ECOG performance status } \\
\hline 0 & $159(62.1)$ \\
\hline 1 & $73(28.5)$ \\
\hline 2 & $24(9.4)$ \\
\hline
\end{tabular}

${ }^{a}$ Differentiation grading and TNM classification for colorectal cancer were based on the 7th American Joint Committee on Cancer TNM staging system (19). ECOG, Eastern Cooperative Oncology Group; TNM, tumor-node-metastasis.

whereas 23 of 25 colorectal adenoma tissues exhibited negative TRAP-1 expression (Fig. 1A).

Cancer recurrence/metastasis according to TRAP-1 expression status. During the follow-up period, cancer recurrences or metastases were detected in 144/256 (56.3\%) patients and at 239 different sites, including anastomotic stoma/pelvic (63 cases), liver (47 cases), lung (34 cases), abdominal cavity/peritoneum (43 cases), regional lymph nodes (26 cases), bone/brain (13 cases) and other unusual sites (13 cases). In these cases, tissues of multiple metastatic sites were detected. The TRAP-1 expression levels in distant metastatic tissues of 22 patients were analyzed, including 11 cases of liver, 6 cases 
A

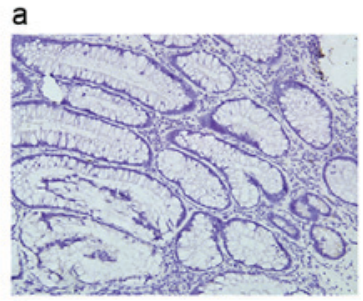

d

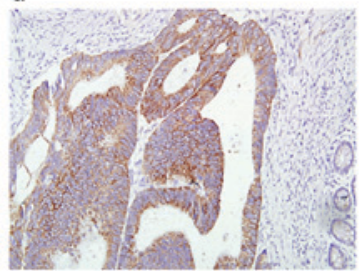

B a

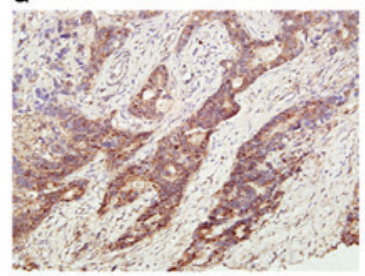

e

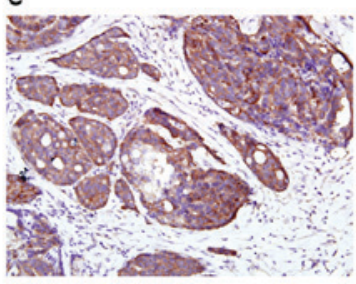

b

f b

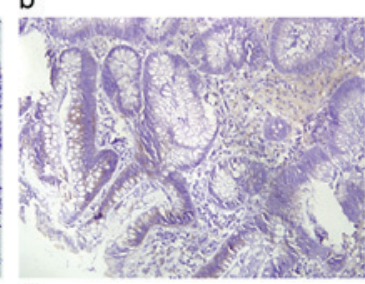

e
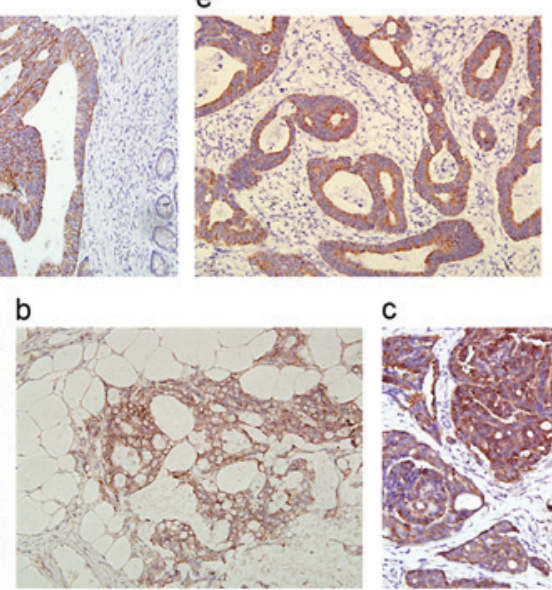

c
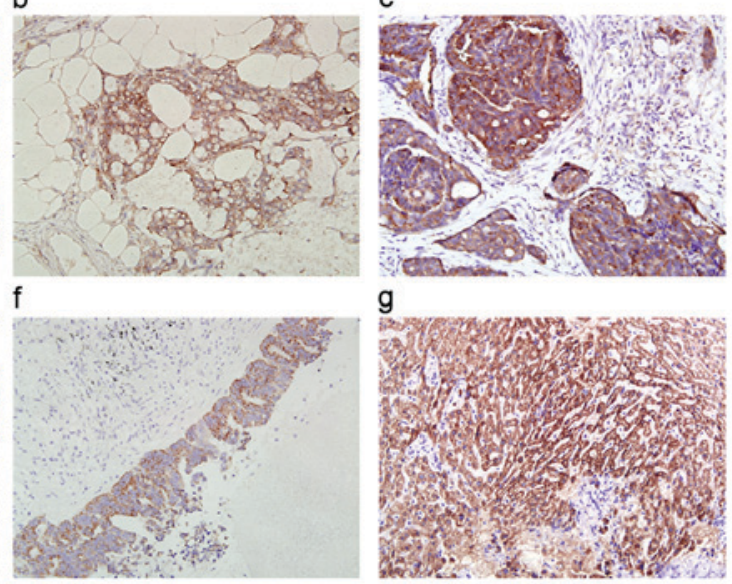

C

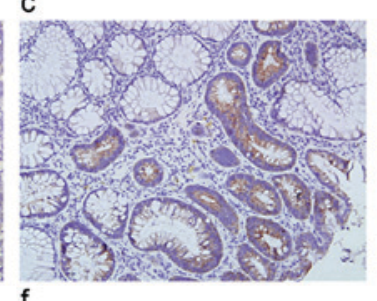

$f$

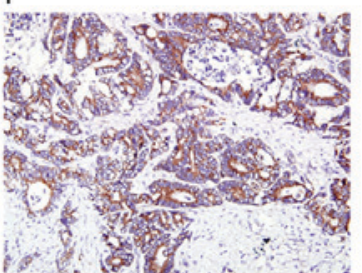

g

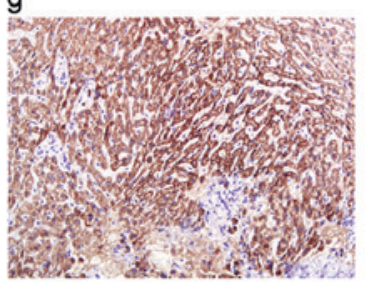

d

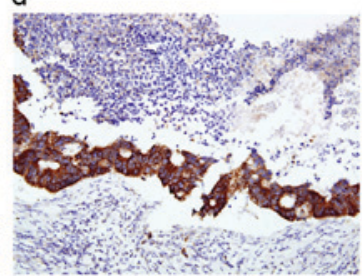

h

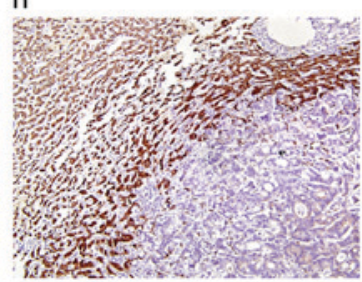

Figure 1. Representative IHC staining of TRAP-1 in colorectal tissues (magnification, x200). (A) IHC staining in differently differentiated CRC tissues: (a) Adjacent non-tumor colorectal tissues with negative TRAP-1 expression; (b) TRAP-1 staining in an exemplary case of colorectal tubular adenoma tissues with negative TRAP-1 expression; (c) TRAP-1 staining in an exemplary case of colorectal high-grade intraepithelial neoplasia tissues with low TRAP-1 expression; (d) well-differentiated CRC tissues with low TRAP-1 expression; (e) moderately differentiated CRC tissues with intermediate TRAP-1 expression; and (f) poorly differentiated CRC tissues with high TRAP-1 expression. (B) IHC staining in CRC tissues at different T stages: (a) T2 stage CRC tissues with low TRAP-1 expression; (b) T3 stage CRC tissues with intermediate TRAP-1 expression; (c) T4 stage CRC tissues with high TRAP-1 expression; (d) TRAP-1 staining in lymph node metastatic CRC tissues with high TRAP-1 expression; (e) TRAP-1 staining in metastatic peritoneum tissues of CRC with high TRAP-1 expression; (f) TRAP-1 staining in metastatic lung tissues of CRC with low TRAP-1 expression and in normal lung tissues with negative TRAP-1 expression; (g) TRAP-1 staining in normal liver tissues with high TRAP-1 expression; (h) TRAP-1 staining in metastatic liver tissues of CRC with low TRAP-1 expression. Grading of differentiation status and TNM classification for CRC were based on the 7th American Joint Committee on Cancer TNM staging system (19). IHC, immunohistochemical; TRAP-1, tumor necrosis factor receptor-associated protein-1; CRC, colorectal cancer.

of lung and 5 cases of peritoneal metastasis. High TRAP-1 expression was determined in all metastatic peritoneal tissues. However, in contrast to peritoneal and lymph node metastases, low TRAP-1 expression was detected in all metastatic liver tissues with respect to normal liver tissues. This indicated an inverse association between TRAP-1 expression levels and liver metastasis. In addition, all metastatic lung tissues exhibited low TRAP-1 expression, in comparison with normal lung tissues, which exhibited negative TRAP-1 expression (Fig. 1B).

Survival analysis according to TRAP-1 expression or expression intensity. Among the 256 patients, 128 mortalities (50.0\%) occurred within 5 years. Kaplan-Meier curves and log-rank tests were applied to determine the association between TRAP-1 expression and 5-year OS and PFS rates, respectively (Fig. 2). As is demonstrated in Fig. 2A and B, TRAP-1 positive expression was significantly associated with decreased 5-year OS $(\mathrm{P}=0.006)$ and PFS $(\mathrm{P}=0.001)$ rates in patients with $\mathrm{CRC}$.
Patients with CRC were analyzed according to high and low TRAP-1 expression groups. Patients with high TRAP-1 expression experienced a 5-year OS rate of $38.0 \%$, compared with $56.5 \%$ for patients with low TRAP-1 expression $(\mathrm{P}=0.003)$. Median OS times were 35.7 and 60.0 months for the high and low TRAP-1 expression groups, respectively. Similarly, the 5-year PFS rate was $26.6 \%$ for patients with high TRAP-1 expression and $53.3 \%$ for patients with low TRAP-1 expression $(\mathrm{P}<0.001)$, and the median PFS times were 21.5 and 60.0 months for the high and low TRAP-1 expression groups, respectively. Patients with CRC with high TRAP-1 expression exhibited shorter OS and PFS times compared with patients with low TRAP-1 expression (Fig. 2C and D).

Prognostic factors associated with TRAP-1 expression status. Univariate analysis demonstrated a significant association between positive TRAP-1 expression and poor OS $(\mathrm{P}=0.008)$ and PFS $(\mathrm{P}=0.001)$ times. Multivariate analyses using Cox's regression model revealed positive TRAP-1 expression as an 
Table II. Association between TRAP-1 expression and clinicopathological features.

\begin{tabular}{|c|c|c|c|c|}
\hline \multirow[b]{2}{*}{ Characteristics } & \multirow[b]{2}{*}{ Total, $\mathrm{n}$} & \multicolumn{2}{|c|}{$\begin{array}{l}\text { Expression intensity of } \\
\text { TRAP- } 1, \mathrm{n}(\%)\end{array}$} & \multirow[b]{2}{*}{ P-value } \\
\hline & & Low & High & \\
\hline All patients & 256 & $179(69.9)$ & $77(30.1)$ & \\
\hline Sex & & & & 0.240 \\
\hline Male & 122 & $81(66.4)$ & $41(33.6)$ & \\
\hline Female & 134 & $98(73.1)$ & $36(26.9)$ & \\
\hline Age, years & & & & 0.605 \\
\hline$<65$ & 140 & $96(68.6)$ & $44(31.4)$ & \\
\hline$\geq 65$ & 116 & $83(71.6)$ & $33(28.4)$ & \\
\hline Tumor location & & & & 0.872 \\
\hline Rectum & 105 & $74(70.5)$ & $31(29.5)$ & \\
\hline Colon & 151 & $105(69.5)$ & $46(30.5)$ & \\
\hline Differentiation $^{\mathrm{a}}$ & & & & 0.011 \\
\hline Well/moderately differentiated & 187 & $139(74.3)$ & $48(25.7)$ & \\
\hline Poorly differentiated & 69 & $40(58.0)$ & $29(42.0)$ & \\
\hline Depth of invasion (pT status) ${ }^{\mathrm{a}}$ & & & & 0.006 \\
\hline $\mathrm{T} 1 / \mathrm{T} 2$ & 32 & $29(90.6)$ & $3(9.4)$ & \\
\hline $\mathrm{T} 3 / \mathrm{T} 4$ & 224 & $150(67.0)$ & $74(33.0)$ & \\
\hline Lymph node metastasis ( $\mathrm{pN}$ status) & & & & $<0.001$ \\
\hline N0 & 139 & $114(82.0)$ & $25(18.0)$ & \\
\hline $\mathrm{N} 1 / \mathrm{N} 2$ & 117 & $65(55.6)$ & $52(44.4)$ & \\
\hline pTNM stage $^{a}$ & & & & $<0.001$ \\
\hline $\mathrm{I} / \mathrm{II}$ & 138 & $107(77.5)$ & $31(22.5)$ & \\
\hline III/IV & 118 & $65(55.1)$ & $53(44.9)$ & \\
\hline
\end{tabular}

${ }^{a}$ Differentiation grading and TNM classification for colorectal cancer were based on the 7th American Joint Committee on Cancer TNM staging system (19). TRAP-1, tumor necrosis factor receptor-associated protein-1; pTNM, pathological tumor-node-metastasis.

independent prognostic factor for both OS [hazard ratio (HR), 1.914; 95\% confidence interval $(\mathrm{CI}), 1.133-3.233 ; \mathrm{P}=0.015]$ and PFS (HR, 2.534; 95\% CI, 1.534-4.212; P<0.001) (Table III).

Furthermore, multivariate analysis demonstrated that an advanced stage, poor Eastern Cooperative Oncology Group (20) performance status, poor tumor differentiation, and the absence of surgery or chemotherapy were independent prognostic factors for cancer-specific OS and PFS times. In addition, no significant association of other factors, such as sex and primary tumor site, with either cancer-specific OS or PFS were identified $(\mathrm{P}>0.05$; Table III).

\section{Discussion}

The present study evaluated the clinical and prognostic significance of TRAP-1 expression in 256 patients with CRC. The results demonstrated that the expression level of TRAP-1 was increased in cancerous tissues compared with adjacent noncancerous tissues, suggesting that TRAP-1 may exhibit a function in CRC tumorigenesis and progression. This result is consistent with reported studies from lung, breast, colorectal and pancreatic adenocarcinomas (15-17). In addition, in the present study, similar percentages of TRAP-1 overexpression were detected in primary colon and rectal cancer tissues $(30.5$ and $29.5 \%$, respectively), indicating that TRAP-1 expression does not differ between colon and rectal cancers.

Clinicopathological parameters serve important functions for predicting prognosis and determining the most suitable CRC therapy (22-24). In the present study, a significant association was demonstrated between an increasing TRAP-1 expression and clinicopathological characteristics of $\mathrm{CRC}$, including differentiation, depth of cancer invasion and presence of lymph node metastasis. Specifically, it was observed that TRAP-1 expression is a predictor for lymph node metastatic spread; this result was in accordance with a previous study of CRC (18) where TRAP-1 positive samples in patients with CRC were more frequently associated with lymphatic metastasis compared with TRAP-1 negative samples. Additionally, TRAP-1 expression was increased in metastatic lymph nodes compared with matched in situ tumor tissues in the present study. Therefore, the association between TRAP-1 expression and the aforementioned clinicopathological factors may implicate TRAP-1 as critical in CRC progression. In turn, TRAP-1 expression may serve as a molecular marker for lymph node metastasis and poor prognosis. However, no significant association was observed between TRAP-1 expression and the clinicopathological parameters of 

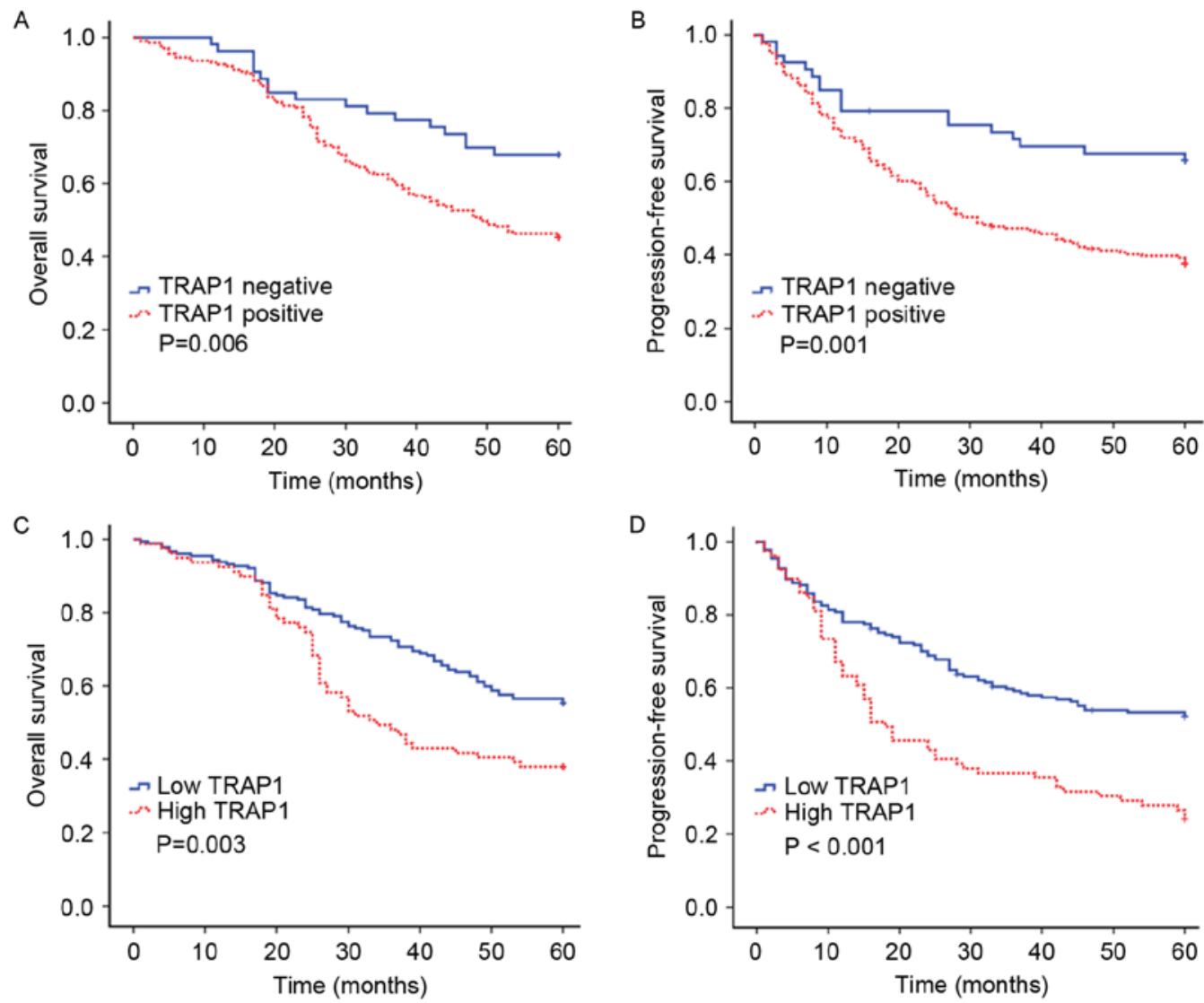

Figure 2. Kaplan-Meier curves (log-rank test) for the correlation of OS and PFS with TRAP-1 expression. (A) OS and (B) PFS according to positive vs. negative TRAP-1 expression. (C) OS and (D) PFS according to high vs. low TRAP-1 expression. TRAP-1, tumor necrosis factor receptor-associated protein-1; OS, overall survival; PFS, progression-free survival.

tumor differentiation and depth of cancer invasion in a previous study (18), and this is inconsistent with the results of the present study. In addition, in the present study no significant difference was indicated between the level of TRAP-1 expression in CRC tissues and other factors, including sex, age and tumor localization $(\mathrm{P}>0.05)$.

However, in the present study, TRAP-1 expression levels from different distant metastatic tissues revealed some contradictory results. Metastatic cancerous tissue of the peritoneum demonstrated an overexpression of TRAP-1, whereas metastatic cancerous tissue of the lung exhibited low expression of TRAP-1. These results suggested that TRAP-1 expression differs between distinct metastatic CRC tissues, and the TRAP-1 expression level in metastatic lung cancer tissues is inconsistent with TRAP-1 expression levels in primary lung tumor tissues (16). Furthermore, TRAP-1 was expressed abundantly in normal liver tissues, whereas TRAP-1 expression was decreased in metastatic liver cancer tissues, indicating that TRAP-1 expression level is inversely associated with metastatic liver cancer.

Notably, TRAP-1 expression levels increased gradually from the colorectal mucosa of high-grade intraepithelial neoplasia to CRC. This suggests that TRAP-1 expression may be detected at the earliest stage of CRC tumorigenesis, and TRAP-1 may serve a function not only in the progression, but also in the onset of malignancy. This suggests that TRAP-1 may be gradually activated during colorectal carcinogenesis. To the best of our knowledge, there were several studies that noted TRAP-1 expression status in colorectal mucosa of high-grade intraepithelial neoplasia. Previous data obtained from CRC associated with ulcerative colitis demonstrated increased TRAP-1 expression and the degree of inflammation in CRC tissues only, but not in acute inflammation tissues (25). These results, in combination with those of the present study, suggest that TRAP-1 expression may occur in the earlier stages of tumorigenesis and that acute inflammation is not likely to influence TRAP-1 expression.

The progression of CRC primarily involves tumor differentiation, local infiltration, lymph node metastasis and distant metastasis. These stages are closely associated with cancer cell proliferation and invasion (26). A number of studies have identified that TRAP-1 expression is upregulated in metastatic cancer cells $(15,22)$ and is involved in oncogenesis by contributing to the inhibition of cancer cell apoptosis $(27,28)$. Other studies have identified that TRAP-1 is abundantly localized in the mitochondria of tumor cells $(13,17)$ and is involved in protecting against oxidative stress and apoptosis (27,29-31). These results demonstrate the anti-apoptotic role of TRAP-1 in cancer cells. In addition, functional studies on the lung adenocarcinoma cell line A549 and the human breast cancer cell line MDA-MB-231 demonstrated that TRAP-1 expression is positively associated with cell proliferation in vitro (28). These significant functions of TRAP-1 in promoting cell proliferation and inhibiting apoptosis may explain the association between TRAP-1 expression levels and clinicopathological characteristics of CRC observed in the present study, including local infiltration, lymph node metastasis and distant metastasis. 
Table III. Univariate and multivariate analyses for OS and PFS of patients with colorectal cancer $(\mathrm{n}=256)$.

\begin{tabular}{|c|c|c|c|c|c|c|}
\hline \multirow[b]{2}{*}{ Variable } & \multicolumn{3}{|c|}{ Univariate analysis } & \multicolumn{3}{|c|}{ Multivariate analysis } \\
\hline & HR & $95 \% \mathrm{CI}$ & P-value & HR & $95 \% \mathrm{CI}$ & P-value \\
\hline \multicolumn{7}{|c|}{ Sex (male vs. female) } \\
\hline OS & 0.815 & $0.576-1.153$ & 0.248 & ND & ND & ND \\
\hline PFS & 0.741 & $0.534-1.028$ & 0.073 & ND & ND & ND \\
\hline \multicolumn{7}{|c|}{ Age $(\geq 65$ vs. $<65$ years $)$} \\
\hline OS & 1.619 & $1.143-2.294$ & 0.007 & 1.022 & $0.700-1.494$ & 0.910 \\
\hline PFS & 1.551 & $1.118-2.152$ & 0.009 & 1.182 & $0.837-1.668$ & 0.342 \\
\hline \multicolumn{7}{|c|}{ Location (rectum vs. colon) } \\
\hline OS & 0.936 & $0.658-1.331$ & 0.712 & ND & ND & ND \\
\hline PFS & 0.932 & $0.668-1.299$ & 0.676 & ND & ND & ND \\
\hline \multicolumn{7}{|c|}{ TNM stage (I/II vs. III/IV) ${ }^{\mathrm{a}}$} \\
\hline OS & 5.144 & $3.491-7.581$ & $<0.001$ & 11.325 & $2.580-49.713$ & 0.001 \\
\hline PFS & 4.215 & 2.963-5.995 & $<0.001$ & 2.511 & $1.661-3.795$ & $<0.001$ \\
\hline \multicolumn{7}{|c|}{ Differentiation (well/moderate vs. poor) ${ }^{\mathrm{a}}$} \\
\hline $\mathrm{OS}$ & 3.777 & $2.656-5.372$ & $<0.001$ & 2.641 & $1.773-3.933$ & $<0.001$ \\
\hline PFS & 3.335 & $2.372-4.690$ & $<0.001$ & 2.219 & $1.514-3.252$ & $<0.001$ \\
\hline \multicolumn{7}{|c|}{ ECOG performance status (0 vs. 1 vs. 2 ) } \\
\hline OS & 3.399 & $2.655-4.352$ & $<0.001$ & 1.591 & $1.169-2.165$ & 0.003 \\
\hline PFS & 2.922 & $2.305-3.705$ & $<0.001$ & 1.585 & $1.171-2.146$ & 0.003 \\
\hline \multicolumn{7}{|c|}{ Surgery (radical surgery vs. palliative surgery) } \\
\hline OS & 0.135 & $0.086-0.212$ & $<0.001$ & 0.345 & $0.202-0.589$ & $<0.001$ \\
\hline PFS & 0.165 & $0.105-0.261$ & $<0.001$ & 0.386 & $0.226-0.658$ & $<0.001$ \\
\hline \multicolumn{7}{|c|}{ Chemotherapy (yes vs. no) } \\
\hline OS & 0.456 & $0.283-0.735$ & 0.001 & 0.323 & $0.192-0.542$ & $<0.001$ \\
\hline PFS & 0.592 & $0.369-0.949$ & 0.030 & 0.548 & $0.35-0.899$ & 0.017 \\
\hline \multicolumn{7}{|c|}{ TRAP-1 expression (negative vs. positive) } \\
\hline OS & 1.997 & $1.198-3.328$ & 0.008 & 1.914 & $1.133-3.233$ & 0.015 \\
\hline PFS & 2.253 & $1.374-3.695$ & 0.001 & 2.534 & $1.534-4.212$ & $<0.001$ \\
\hline
\end{tabular}

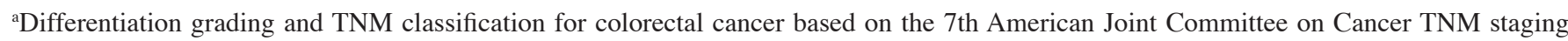
system (19). OS, overall survival; PFS; progression-free survival; HR, hazard ratio; CI, confidence interval; ECOG, Eastern Cooperative Oncology Group; TNM, tumor-node-metastasis; ND, not determined; TRAP-1, tumor necrosis factor receptor-associated protein-1.

A number of previous studies have demonstrated that the main roles of TRAP-1 are in tumor progression, protection from oxidative damage and cell survival $(9,29)$. TRAP-1 interacts with CypD, a mitochondrial permeability transition pore regulator, to suppress the main cell death pathway maintained by CypD $(10,27)$. Therefore, cytoprotection mediated by the upregulated expression of TRAP-1 may be an indicator for the onset of tumorigenesis. Furthermore, it has been reported that TRAP-1 functions synergistically with tumor necrosis factor receptor 1 to modulate the expression of the cell adhesion molecule $\mathrm{N}$-cadherin, while altering the inter-cellular adhesion of neuronal cells through the signal transducer and activator of transcription 3 phosphorylation status $(17,32)$. This demonstrates the role of TRAP-1 in the processes of cell invasion and motility, which are characteristics of tumorigenesis and metastatic spread. In addition, previous studies have demonstrated that TRAP-1 regulates genes involved in the cell cycle and metastasis (28). The present study also indicated that cytoprotective mitochondrial chaperone TRAP-1 may be viewed as a molecular target in localized and metastatic CRC.

In the present study, follow-up analysis revealed that TRAP-1 expression was associated with cancer-specific 5-year OS and PFS rates in 256 patients with CRC, independent of the degree of TRAP-1 expression. Patients with positive or high TRAP-1 expression experienced poorer rates of cancer-specific 5-year OS and PFS compared with patients with negative or low TRAP-1 expression. Univariate and multivariate analyses revealed that TRAP-1 expression is an independent prognostic factor for cancer-specific OS and PFS of CRC. These observations support previous studies suggesting that overexpression of TRAP-1 is associated with a shortened OS time in other types of cancer, including breast, lung and prostate cancer (15-17).

A major limitation of the present study is the limited number of cases $(n=256)$. A study on an increased scale is required to confirm the clinical significance of TRAP-1 expression. Due to the multiple roles of TRAP-1 in cellular functioning, future 
research is required to elucidate the mechanisms underlying TRAP-1 expression and its role in cancer cell biology.

In conclusion, the present study demonstrated the association between TRAP-1 expression and cancer-specific OS and PFS of human CRC. As well as this, overexpression of TRAP-1 was identified to be an adverse prognostic factor for patients with CRC. Further studies on an increased scale will be required to validate these results.

\section{Acknowledgements}

The authors wish to thank Dr Li-Jiang Liu (JiangHan University Pathology Center, Jianghan University of China, Wuhan, China) and Li-Hua Huang (Department of Pathology, PuAi Hospital of Tongji Medical College, Huazhong University of Science and Technology of Wuhan, Wuhan, China) for their technical assistance. The present study was supported by the Natural Science Foundation of Hubei (grant nos. 2012FFB05601 and 2016CFB340), Wuhan Yellow Crane Medical Talent Program, Wuhan Science and Technology Bureau (grant nos. 201260523197, 201271130460, 2013060602010259 and 2015061701011626) and National Natural Science Foundation of China (grant no. 81502461).

\section{References}

1. Siegel R, Desantis C and Jemal A: Colorectal cancer statistics, 2014. CA Cancer J Clin 64: 104-117, 2014.

2. Moniuszko T, Wincewicz A, Koda M, Domysławska I and Sulkowski S: Role of periostin in esophageal, gastric and colon cancer. Oncol Lett 12: 783-787, 2016.

3. Rogers AC, Winter DC, Heeney A, Gibbons D, Lugli A, Puppa G and Sheahan K: Systematic review and meta-analysis of the impact of tumour budding in colorectal cancer. Br J Cancer 115 831-840, 2016.

4. Bond A, O'Toole P, Fisher G, Subramanian S, Haslam N, Probert C, Cox T and Sarkar S: New-generation high-definition colonoscopes increase adenoma detection when screening a moderate-risk population for colorectal cancer. Clin Colrectal Cancer 16: 44-50, 2017.

5. Chand M, Rasheed S, Heald R, Swift I, West N, Rao S, Tekkis P and Brown G: Adjuvant chemotherapy may improve disease-free survival in patients with persistently mrEMVI-positive rectal cancer following chemoradiation. Colorectal Dis 19: 537-543, 2017.

6. Yuan D, Li K, Zhu K, Yan R and Dang C: Plasma miR-183 predicts recurrence and prognosis in patients with colorectal cancer. Cancer Biol Ther 16: 268-275, 2015.

7. Torre LA, Bray F, Siegel RL, Ferlay J, Lortet-Tieulent J and Jemal A: Global cancer statistics, 2012. CA Cancer J Clin 65: 87-108, 2015

8. Simon K: Colorectal cancer development and advances in screening. Clin Interv Aging 11: 967-976, 2016.

9. Matassa DS, Amoroso MR, Maddalena F, Landriscina M and Esposito F: New insights into TRAP1 pathway. Am J Cancer Res 2: 235-248, 2012.

10. Neckers L and Ivy SP: Heat shock protein 90. Curr Opin Oncol 5: 419-424, 2003.

11. Lianos GD, Alexiou GA, Mangano A, Mangano A, Rausei S, Boni L, Dionigi G and Roukos DH: The role of heat shock proteins in cancer. Cancer Lett 360: 114-118, 2015.

12. Hua G, Zhang Q and Fan Z: Heat shock protein 75 (TRAP1) antagonizes reactive oxygen species generation and protects cells from granzyme M-mediated apoptosis. J Biol Chem 282: 20553-20560, 2007.

13. Kang BH, Plescia J, Dohi T, Rosa J, Doxsey SJ and Altieri DC: Regulation of tumor cell mitochondrial homeostasis by an organelle-specific Hsp90 chaperone network. Cell 131: 257-270, 2007.
14. Tian X, Ma P, Sui CG, Meng FD, Li Y, Fu LY, Jiang T, Wang Y and Jiang YH: Suppression of tumor necrosis factor receptor-associated protein 1 expression induces inhibition of cell proliferation and tumor growth in human esophageal cancer cells. FEBS J 281: 2805-2819, 2014.

15. Zhang B, Wang J, Huang Z, Wei P, Liu Y, Hao J, Zhao L, Zhang F, $\mathrm{Tu} \mathrm{Y}$ and Wei T: Aberrantly upregulated TRAP1 is required for tumorigenesis of breast cancer. Oncotarget 6: 44495-44508, 2015.

16. Agorreta J, Hu J, Liu D, Delia D, Turley H, Ferguson DJ, Iborra F, Pajares MJ, Larrayoz M, Zudaire I et al: TRAP1 regulates proliferation, mitochondrial function, and has prognostic significance in NSCLC. Mol Cancer Res 12: 660-669, 2014.

17. Leav I, Plescia J, Goel HL, Li J, Jiang Z, Cohen RJ, Languino LR and Altieri DC: Cytoprotective mitochondrial chaperone TRAP-1 as a novel molecular target in localized and metastatic prostate cancer. Am J Pathol 176: 393-401, 2010.

18. Gao JY, Song BR, Peng JJ and Lu YM: Correlation between mitochondrial TRAP-1 expression and lymph node metastasis in colorectal cancer. World J Gastroenterol 18: 5965-5971, 2012.

19. Rusch VW, Rice TW, Crowley J, Blackstone EH, Rami-Porta R and Goldstraw P: The seventh edition of the American Joint Committee on Cancer/International Union Against Cancer Staging Manuals: The new era of data-driven revisions. J Thorac Cardiovasc Surg 139: 819-821, 2010.

20. Hofheinz RD, Ronellenfitsch U, Kubicka S, Falcone A, Burkholder I and Hacker UT: Treatment with antiangiogenic drugs in multiple lines in patients with metastatic colorectal cancer: Meta-analysis of randomized trials. Gastroenterol Res Pract 2016: 29189483, 2016.

21. Ou Y, Liu L, Xue L, Zhou W, Zhao Z, Xu B, Song Y and Zhan Q: TRAP1 shows clinical significance and promotes cellular migration and invasion through STAT3/MMP2 pathway in human esophageal squamous cell cancer. J Genet Genomics 41: 529-537, 2014.

22. Khanna A, Böckelman C, Hemmes A, Junttila MR, Wiksten JP, Lundin M, Junnila S, Murphy DJ, Evan GI, Haglund C et al: MYC-dependent regulation and prognostic role of CIP2A in gastric cancer. J Natl Cancer Inst 101: 793-805, 2009.

23. Elias E, Mukherji D, Faraj W, Khalife M, Dimassi H, Eloubeidi M, Hattoum H, Abou-Alfa GK, Saleh A and Shamseddine A: Lymph-node ratio is an independent prognostic factor in patients with stage III colorectal cancer: A retrospective study from the Middle East. World J Surg Oncol 10: 63, 2012.

24. Chew MH, Teo JY, Kabir T, Koh PK, Eu KW and Tang CL: Stage IV colorectal cancers: An analysis of factors predicting outcome and survival in 728 cases. J Gastrointest Surg 16: 603-612, 2012.

25. Chen R, Pan S, Lai K, Lai LA, Crispin DA, Bronner MP and Brentnall TA: Up-regulation of mitochondrial chaperone TRAP1 in ulcerative colitis associated colorectal cancer. World J Gastroenterol 20: 17037-17048, 2014.

26. Hauptman N and Glavač D: Colorectal cancer blood-based biomarkers. Gastroenterol Res Pract 2017: 22195361, 2017.

27. Montesano Gesualdi N, Chirico G, Pirozzi G, Costantino E, Landriscina $\mathrm{M}$ and Esposito F: Tumor necrosis factor-associated protein 1 (TRAP-1) protects cells from oxidative stress and apoptosis. Stress 10: 342-350, 2007.

28. Nakagawa T, Shimizu S, Watanabe T, Yamaguchi O, Otsu K, Yamagata H, Inohara H, Kubo T and Tsujimoto Y: Cyclophilin D-dependent mitochondrial permeability transition regulates some necrotic but not apoptotic cell death. Nature 434: 652-658, 2005.

29. Liu D, Hu J, Agorreta J, Cesario A, Zhang Y, Harris AL, Gatter K and Pezzella F: Tumor necrosis factor receptor-associated protein 1(TRAP1) regulates genes involved in cell cycle and metastases. Cancer Lett 296: 194-205, 2010.

30. Landriscina M, Laudiero G, Maddalena F, Amoroso MR, Piscazzi A, Cozzolino F, Monti M, Garbi C, Fersini A, Pucci P and Esposito F: Mitochondrial chaperone Trap1 and the calcium binding protein Sorcin interact and protect cells against apoptosis induced by antiblastic agents. Cancer Res 70: 6577-6586, 2010.

31. Guzzo G, Sciacovelli M, Bernardi P and Rasola A: Inhibition of succinate dehydrogenase by the mitochondrial chaperone TRAP1 has anti-oxidant and anti-apoptotic effects on tumor cells. Oncotarget 5: 11897-11908, 2014.

32. Kubota $\mathrm{K}$, Inoue $\mathrm{K}$, Hashimoto R, Kumamoto N, Kosuga A, Tatsumi M, Kamijima K, Kunugi H, Iwata N, Ozaki N, et al: Tumor necrosis factor receptor-associated protein 1 regulates cell adhesion and synaptic morphology via modulation of $\mathrm{N}$-cadherin expression. J Neurochem 110: 496-508, 2009.

This work is licensed under a Creative Commons Attribution-NonCommercial-NoDerivatives 4.0 International (CC BY-NC-ND 4.0) License. 